Check for updates

Cite this: RSC Adv., 2018, 8, 25941

Received 5th June 2018

Accepted 10th July 2018

DOI: $10.1039 / \mathrm{c} 8 \mathrm{ra0} 4778 \mathrm{~b}$

rsc.li/rsc-advances

\section{Photostability and antioxidant activity studies on the inclusion complexes of trans-polydatin with $\beta$ - cyclodextrin and derivatives $\dagger$}

\author{
Shujing Li, (D)*ab Li Yuan, ${ }^{\text {ab }}$ Bing Zhang, *c Wei Zhou, ${ }^{\text {ab }}$ Xinrui Wang ${ }^{\text {ab }}$ \\ and Dongsheng Bai ${ }^{\mathrm{ab}}$
}

The inclusion complexes of trans-polydatin and three cyclodextrins (CDs), namely $\beta$-cyclodextrin ( $\beta$-CD), methyl- $\beta$-cyclodextrin (Me- $\beta$-CD) and (2-hydroxy) propyl- $\beta$-cyclodextrin (HP- $\beta-C D$ ) were prepared. The effects of the inclusion behavior of trans-polydatin with three kinds of CDs were investigated in both solution and the solid state with the following methods: phase-solubility, X-ray diffraction (XRD), thermogravimetric analysis (TG), differential scanning calorimetry (DSC), and scanning electron microscopy (SEM), proton nuclear magnetic resonance $\left({ }^{1} \mathrm{H}-\mathrm{NMR}\right)$ and two-dimensional rotational frame nuclear overhauser effect spectroscopy (2D ROESY). The results indicated that trans-polydatin formed a $1: 1$ stoichiometric inclusion complex with CDs. Meanwhile, the solubility and thermal stability of the inclusion complexes were improved after encapsulating by CDs. Furthermore, the photostability of trans-polydatin was enhanced after forming the inclusion complexes. The antioxidant activities results showed that the antioxidant performance of the inclusion complexes was enhanced in comparison to the native trans-polydatin. Therefore, it can be a potentially promising way to promote its drug bioavailability or phytochemical preparations.

\section{Introduction}

Polydatin $\quad\left(3,4^{\prime}, 5\right.$-trihydroxystilbene-3- $\beta$-mono-D-glucoside, Fig. 1a) is the major component of Polygonum cuspidatum root, and is widely used in medicine and health products. ${ }^{1-3}$ Polydatin has been shown to possess biological activities, which include anti-platelet aggregation, ${ }^{4}$ anti-cancer effects, ${ }^{5}$ antioxidative activities, ${ }^{6,7}$ and anti-inflammatory activity. ${ }^{8}$ It also has multiple activities in the cardiovascular and hematological system and can protect myocardial cells injured by deprivation of oxygen and glucose.9,10 Even though polydatin has a vast range of health benefits, the practical biological application of polydatin is limited due to its poor water solubility and stability. ${ }^{\mathbf{1 1}}$ Interestingly, these problems can be solved by complexation with cyclodextrins (CDs) in aqueous solutions. Cyclodextrins (CDs) are cyclic oligosaccharides consisting of ( $\alpha$ 1,4)-linked glucopyranose units with a hydrophobic cavity and a hydrophilic outer surface. ${ }^{\mathbf{1 2}}$ The truncated cone-shaped

${ }^{a}$ Beijing Advanced Innovation Center for Food Nutrition and Human Health, Beijing Technology and Business University, Beijing 100048, PR China. E-mail: lishujing@ th.btbu.edu.cn

${ }^{b}$ Department of Chemistry, School of Science, Beijing Technology and Business University, Beijing 100048, PR China

${ }^{c}$ Technical Institute of Physics and Chemistry, Chinese Academy of Science, Beijing 100190, PR China

$\dagger$ Electronic supplementary information (ESI) available. See DOI: $10.1039 / \mathrm{c} 8 \mathrm{ra} 04778 \mathrm{~b}$ structure of CDs enables them to enclose the hydrophobic molecules to form the inclusion complex..$^{13-17}$ The CDs and their inclusion complexes are always used as delivery systems in the drug, food, cosmetics, packaging, and textile industries. ${ }^{18,19} \beta$ Cyclodextrin ( $\beta$-CD) is the most commonly used in pharmaceutical formulations due to its non-toxicity, biodegradability and reasonable cost. However, the application of unmodified $\beta$ $\mathrm{CD}$ is limited owing to its poor water solubility. Therefore, modified $\beta$-CDs have been synthesized and used, such as methylated- $\beta$-cyclodextrin (Me- $\beta$-CD) and (2-hydroxy) propyl- $\beta$ cyclodextrin (HP- $\beta$-CD). ${ }^{\mathbf{2 0 , 2 1}}$

Recently, An et al. investigated the inclusion behavior of HP$\beta$-CD with polydatin in solution and its analytical application. ${ }^{22}$ (a)<smiles>OCC1OC(Oc2cc(O)cc(/C=C/c3ccc(O)cc3)c2)[C@H](O)[C@@H](O)[C@@H]1O</smiles>

(b)<smiles>OC[C@H]1O[C@@H](Oc2cc(O)cc(/C=C\c3ccc(O)cc3)c2)[C@H](O)[C@@H](O)[C@@H]1O</smiles>

Fig. 1 Chemical structure of trans-polydatin (a) and cis-polydatin (b). 
Zhang and Liu reported the inclusion complex of polydatin with $\beta$-CD, $\gamma$-CD and 6- $O-\alpha$-maltosyl- $\beta-\mathrm{CD}$, their physicochemical characteristics has been studied and characterized. ${ }^{23,24}$ Although these studies have demonstrated the feasibility of obtaining inclusion complexes with polydatin, none of them evaluated the photostability and antioxidant activities of polydatin with different CDs. Polydatin exists in two isomeric forms, trans-polydatin (Fig. 1a) and cis-polydatin (Fig. 1b); the former has been implicated as a bioactive material of major benefit to humans. However trans-polydatin is highly unstable to UV light and readily isomerizes to the cis-polydatin form. In this work we are aimed to overcome the isomerization of the highly valuable trans-polydatin by using the extraordinary properties of CDs to form host-guest inclusion complexes and evaluate the antioxidant activities of the inclusion complexes. The inclusion complexes were prepared by the freeze-drying method and were further characterized by XRD, TG, DSC, SEM, ${ }^{1} \mathrm{H}-\mathrm{NMR}$ and $2 \mathrm{D}$ ROESY. The photostability of trans-polydatin before and after encapsulating by CDs were characterized by UV-vis spectra. Meanwhile, the antioxidant activities of trans-polydatin and the inclusion complexes were also investigated by the 1,1-diphenyl2-picrylhydrazyl (DPPH) radical scavenging activity assay.

\section{Experimental}

\section{Materials}

trans-Polydatin $(>99 \%)$, potassium ferricyanide $\left(\mathrm{K}_{3} \mathrm{Fe}(\mathrm{CN})_{6}\right)$, trichloroacetic acid (TCA), ferric chloride $\left(\mathrm{FeCl}_{3}\right)$ were obtained from Aladdin Industrial Corporation (Shanghai, China); $\beta$ cyclodextrin ( $\beta$-CD, $M_{\mathrm{w}}=1135 \mathrm{~g} \mathrm{~mol}^{-1}$ ), methyl- $\beta$-cyclodextrin $\left(\mathrm{Me}-\beta-\mathrm{CD}, M_{\mathrm{w}}=1310 \mathrm{~g} \mathrm{~mol}^{-1}\right)$, average degree of substitution (DS $=12.5)$, (2-hydroxy) propyl- $\beta$-cyclodextrin (HP- $\beta$-CD, $M_{\mathrm{w}}=$ $1375 \mathrm{~g} \mathrm{~mol}^{-1}$ ), average degree of substitution (DS $=4.2$ ) were purchased from Seebio Biotech, Inc. (Shanghai, China); 1,1diphenyl-2-picryl-hydrazyl (DPPH) was supplied by TCI (Shanghai) Development Co., Ltd.

\section{Preparation of trans-polydatin- $\beta$-CD, trans-polydatin-Me- $\beta$ - $\mathrm{CD}$ and trans-polydatin-HP- $\beta-\mathrm{CD}$ inclusion complexes}

trans-Polydatin ( $0.3 \mathrm{mmol}$ ) was dissolved in $40 \mathrm{~mL}$ ethanol, CDs ( $\beta$-CD, Me- $\beta$-CD, HP- $\beta$-CD, $0.3 \mathrm{mmol}$ ) were dissolved in $160 \mathrm{~mL}$ water, and then the CDs solutions were added to the transpolydatin solution. The mixture was sealed and stirred for $48 \mathrm{~h}$. After evaporating the ethanol from the reaction mixture, the uncomplexed trans-polydatin was filtered through a $0.45 \mu \mathrm{m}$ hydrophilic membrane filter. The filtrate was frozen at $-40{ }^{\circ} \mathrm{C}$ for $24 \mathrm{~h}$ and then lyophilized. The resultant powers collected as the trans-polydatin-CDs complexes.

The encapsulation efficiency of trans-polydatin was determined with the modified procedure descried by Li et al. ${ }^{25}$ In brief, the trans-polydatin-CDs inclusion complex was dispersed in $50 \%$ methanol solution and incubated at $60^{\circ} \mathrm{C}$ for $1 \mathrm{~h}$. After filtration, the absorbance of filtrate was determined by UV-vis spectrum to calculate the included trans-polydatin in the inclusion complex. The encapsulation efficiency $\left(E_{\mathrm{r}}\right)$ were obtained using following equations:

$$
E_{\mathrm{r}}=\frac{M_{\mathrm{i}}}{M_{\mathrm{t}}} \times 100 \%
$$

where, $M_{\mathrm{i}}$ is the included trans-polydatin content in the inclusion complex, and $M_{\mathrm{t}}$ is the total trans-polydatin content in reaction system.

The $E_{\mathrm{r}}$ of trans-polydatin- $\beta$-CD was calculated to be $74.83 \pm$ $1.92 \%$. The $E_{\mathrm{r}}$ of trans-polydatin-HP- $\beta$-CD was calculated to be $77.31 \pm 2.41 \%$. The $E_{\mathrm{r}}$ of trans-polydatin-Me- $\beta$-CD was calculated to be $79.12 \pm 1.67 \%$.

\section{Preparation of trans-polydatin/ $\beta$-CD, trans-polydatin/Me- $\beta$ - CD and trans-polydatin/HP- $\beta$-CD physical mixture}

The physical mixture was prepared by mixing the powders in a $1: 1$ molar ratio of polydatin and CDs in an agate mortar.

\section{Phase-solubility study}

Phase-solubility studies were performed according to the method reported by Higuchi and Connors. ${ }^{26}$ An excess amount of trans-polydatin was added to $10 \mathrm{~mL}$ of aqueous solution containing different concentrations of $\beta-\mathrm{CD}$, Me- $\beta-\mathrm{CD}$, and HP$\beta$-CD (from $0 \mathrm{mM}$ to $10.0 \mathrm{mM}$ ). The mixtures were vigorously shaken with a shaking rate at $120 \mathrm{rpm}$ in water bath for $72 \mathrm{~h}$ at $25{ }^{\circ} \mathrm{C}$. After reaching equilibrium, the samples were filtered through a $0.45 \mu \mathrm{m}$ hydrophilic membrane filter. All samples were prepared in triplicate. The concentration of trans-polydatin in the filtrate was determined by a CARY-60 spectrophotometer (Varian, America). The phase-solubility profiles were obtained by plotting the solubility of trans-polydatin $v s$. the concentration of $\beta-\mathrm{CD}$, Me- $\beta-\mathrm{CD}$, or HP- $\beta$-CD. The apparent stability constants $\left(K_{\mathrm{S}}\right)$ were calculated from phase-solubility diagrams according to the following equation:

$$
K_{\mathrm{s}}=\frac{\text { Slope }}{S_{0}(1-\text { Slope })}
$$

where $S_{0}$ is the solubility of trans-polydatin at $25{ }^{\circ} \mathrm{C}$ in the absence of cyclodextrins and slope means the corresponding slope of the phase-solubility diagrams.

\section{Powder X-ray diffraction (XRD)}

Monochromatic $\mathrm{Cu} \mathrm{K} \alpha$ radiation (wavelength $=1.54056 \AA$ ) was produced by D/MAX 2500V/PC X-ray diffractometer (Rigaku Americas Corporation, Japan). The powders of samples were packed tightly in a rectangular aluminum cell. The samples were exposed to the X-ray beam. The scanning regions of the diffraction angle, $2 \theta$, were $5-50^{\circ}$. Duplicate measurements were made at ambient temperature. Radiation was detected with a proportional detector.

\section{Thermal analyses (TG) and differential scanning calorimetry (DSC)}

TG and DSC measurements were performed with a DTG-60AH (Shimadzu, Japan) instrument, at a heating rate of $10{ }^{\circ} \mathrm{C} \min ^{-1}$ from $30{ }^{\circ} \mathrm{C}$ to $400{ }^{\circ} \mathrm{C}$ in a dynamic nitrogen atmosphere (flow rate $=70 \mathrm{~mL} \mathrm{~min}^{-1}$ ). 


\section{Scanning electron microscopy (SEM)}

SEM photographs were determined on a TESCAN VEGA II. The powders were previously fixed on a brass stub using doublesided adhesive tape and then were made electrically conductive by coating, in a vacuum with a thin layer of gold for $30 \mathrm{~s}$ and at $20 \mathrm{~W}$.

\section{${ }^{1}$ H-NMR and 2D NMR}

The ${ }^{1} \mathrm{H}-\mathrm{NMR}$ and $2 \mathrm{D}$ ROESY spectra were all recorded on a BRUKER AVANCE $600 \mathrm{NMR}$ spectrometer at $25^{\circ} \mathrm{C}$. Deuterium oxide $\left(\mathrm{D}_{2} \mathrm{O}\right)$ was used as the solvent. Chemical shifts were referenced to the solvent values (4.70 $\mathrm{ppm}$ for HOD).

\section{Photostability study of trans-polydatin and inclusion complexes}

The native trans-polydatin and trans-polydatin-CDs inclusion complexes $\left(6 \times 10^{-5} \mathrm{~mol} \mathrm{~L}^{-1}\right)$ were used for UV irradiation analysis. Aliquots of $20 \mathrm{~mL}$ were added to a $6 \mathrm{~cm}$ diameter Petri dish and placed $20 \mathrm{~cm}$ from the UV light source. The photo intensity is $21.0 \mathrm{~W} \mathrm{~cm}^{-2}$. The solutions were irradiated at $25^{\circ} \mathrm{C}$ for $1.5-2 \mathrm{~h}$ and monitored by UV-vis spectrophotometer.

\section{Antioxidant and free-radical scavenging activities}

Reducing power assay. The reducing power (RP) of the transpolydatin-CDs inclusion complexes and native trans-polydatin were investigated according to the method of Ma et al. ${ }^{27}$ The different concentrations of the samples with $2.0 \mathrm{~mL}$ of phosphate buffer $(0.2 \mathrm{M}, \mathrm{pH} 6.6)$ and $2.0 \mathrm{~mL}$ of $\mathrm{K}_{3} \mathrm{Fe}(\mathrm{CN})_{6}(1 \%, \mathrm{w} / \mathrm{v})$. In the next step, the mixtures were incubated at $50{ }^{\circ} \mathrm{C}$ for $20 \mathrm{~min}$, and then rapidly cooled. $2.0 \mathrm{~mL}$ of TCA solution $(10.0 \%$, $\mathrm{w} / \mathrm{v}$ ) was added to the mixture. $2.0 \mathrm{~mL}$ of upper layer of the solution was taken out and mixed with $2.0 \mathrm{~mL}$ of deionized water and $0.4 \mathrm{~mL}$ of $\mathrm{FeCl}_{3}(0.1 \% \mathrm{w} / \mathrm{v})$. The absorbance after $10 \mathrm{~min}$ reaction was measured using an UV-vis spectrophotometer at $700 \mathrm{~nm}$.

DPPH radical-scavenging capacity. DPPH free-radical scavenging by the trans-polydatin-CDs inclusion complexes and native trans-polydatin were investigated according to the method of Hatano et al. ${ }^{28}$ Briefly, DPPH solutions $(2.0 \mathrm{~mL})$ in ethanol $\left(1 \times 10^{-4} \mathrm{~mol} \mathrm{~L}^{-1}\right)$ and $2.0 \mathrm{~mL}$ of tested samples with various concentrations were mixed in the tubes. Then, the mixture was incubated for $60 \mathrm{~min}$ in the dark at $30 \pm 1{ }^{\circ} \mathrm{C}$. The absorbance was measured at $517 \mathrm{~nm}$ in CARY-60 UV-vis spectrometer. Lower absorbance of the reaction mixture indicated higher free radical scavenging activity. The DPPH scavenging effect (KD) was calculated using the following equation:

$$
K_{\mathrm{D}}=\left(\frac{A_{0}-\left(A_{\mathrm{i}}-A_{\mathrm{j}}\right)}{A_{0}}\right) \times 100 \%
$$

where $A_{0}$ is the absorbance of $2.0 \mathrm{~mL} \mathrm{DPPH}$ solution and $2.0 \mathrm{~mL}$ sample solvent, $A_{\mathrm{i}}$ is the absorbance of $2.0 \mathrm{~mL} \mathrm{DPPH}$ solution and $2.0 \mathrm{~mL}$ sample solution and $A_{\mathrm{j}}$ is the absorbance of $2.0 \mathrm{~mL}$ sample solution and $2.0 \mathrm{~mL}$ ethanol.
Statistical analysis. All analytical experiments were carried out in three replicates and the results presented as a mean \pm standard deviation (SD). Analysis of variance (ANOVA) procedure followed by Duncan's test using SPSS 13 (SPSS Inc., Chicago, Illinois, USA) software.

\section{Results and discussion}

\section{Phase-solubility study}

Phase-solubility analysis of trans-polydatin with $\beta$-CD, Me- $\beta$-CD, and HP- $\beta-C D$ was performed by the method of Higuchi and Connors in aqueous solution at $25{ }^{\circ} \mathrm{C}^{26}$ The phase-solubility diagram is a widely useful technique for evaluation of the inclusion effect of CDs with poor water-soluble molecules and determination of the stability constants $\left(K_{\mathrm{s}}\right)$. As shown in Fig. 2, the aqueous solubility of trans-polydatin increased linearly with increasing CDs concentration within the studied concentration range. Based on Higuchi and Connors's theory, these three linear host-guest correlations can be classified as $A_{\mathrm{L}}$ type, indicating that a $1: 1$ stoichiometry of the complexes between trans-polydatin and the three different CDs studied. The calculated apparent stability constant $\left(K_{\mathrm{s}}, \mathrm{M}^{-1}\right)$ of trans-polydatin- $\beta$-CD, trans-polydatin-Me- $\beta$-CD and trans-polydatin-HP- $\beta$ $\mathrm{CD}$, was $798 \mathrm{M}^{-1}, 1106 \mathrm{M}^{-1}$, and $1308 \mathrm{M}^{-1}$, respectively. The higher apparent stability constant of trans-polydatin-Me- $\beta$-CD and trans-polydatin-HP- $\beta-\mathrm{CD}$ can be attributed to the opening enlargement of native $\beta-\mathrm{CD}$ and destruction of the strong intramolecular hydrogen bond network by the methyl and hydroxypropyl substitutions which lets guest molecules access the CD cavity easily and give a higher stability constant. The findings states that CDs increase the binding capacity for flavonoids have been previously reported. ${ }^{29,30}$ Additionally, the solubility of trans-polydatin was significantly increased (6.4fold, 7.9-fold, and 9.0-fold at $10 \mathrm{mM}$ of $\beta-\mathrm{CD}$, Me- $\beta-\mathrm{CD}$, and HP$\beta$-CD) compared to the absence of CDs, which indicated the solubilizing potential for trans-polydatin by CDs.

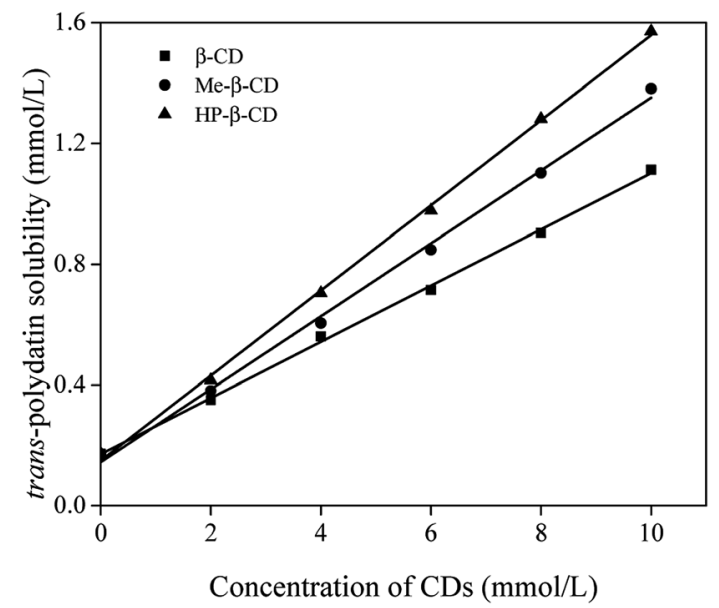

Fig. 2 Phase-solubility diagrams of trans-polydatin with $\beta-C D, M e-\beta$ $\mathrm{CD}$, or $\mathrm{HP}-\beta-\mathrm{CD}$ at $25^{\circ} \mathrm{C}$. 

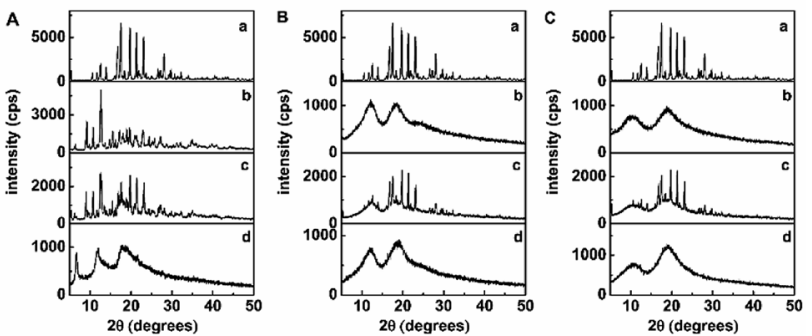

Fig. 3 XRD patterns: (A) (a) trans-polydatin, (b) $\beta$-CD, (c) trans-polydatin/ $\beta-C D$ physical mixture, (d) trans-polydatin- $\beta-C D$ inclusion complex; (B) (a) trans-polydatin, (b) Me- $\beta-C D$, (c) trans-polydatin/Me$\beta-C D$ physical mixture, (d) trans-polydatin-Me- $\beta-C D$ inclusion complex; (C) (a) trans-polydatin, (b) HP- $\beta-C D$, (c) trans-polydatin/HP$\beta-C D$ physical mixture, (d) trans-polydatin-HP- $\beta-C D$ inclusion complex.

\section{XRD studies}

XRD is an effective method for the analysis of CDs and their inclusion complex in the powder or microcrystalline state. ${ }^{31,32}$ The formation of an inclusion complex between cyclodextrin and a crystalline guest mean that the latter would no longer exist in the crystalline state and, consequently, the diffraction pattern of the complex would not be a simple superposition of those two components. As indicated in Fig. 3, the XRD patterns of trans-polydatin and $\beta$-CD displayed numerous sharp peaks characteristic of its crystallinity, whereas that of Me- $\beta-\mathrm{CD}$ and HP- $\beta$-CD showed two broad peaks, consistent with its amorphous nature. The XRD of the physical mixture of trans-polydatin and CDs was a superposition of the patterns of the components, confirming that no chemical association was occurred between trans-polydatin and CDs, and both kept their original physical characteristics. In contrast, XRD of trans-polydatin- $\beta-\mathrm{CD}$, trans-polydatin-Me- $\beta-\mathrm{CD}$, and trans-polydatin-HP$\beta$-CD inclusion complex are amorphous and show halo patterns, indicating the interaction between trans-polydatin and $\beta-\mathrm{CD}(\mathrm{Me}-\beta-\mathrm{CD}$, or HP- $\beta-\mathrm{CD})$.

\section{Thermal analysis}

The thermal properties of trans-polydatin, CDs, and transpolydatin-CDs inclusion complexes were studied by TG methods $^{33}$ (see ESI Fig. S1-S3†). A systematic analysis of the TG curves showed that trans-polydatin decomposes at ca. $270{ }^{\circ} \mathrm{C}, \beta$ $\mathrm{CD}$ at ca. $298{ }^{\circ} \mathrm{C}$, Me- $\beta$-CD at ca. $290{ }^{\circ} \mathrm{C}$, and HP- $\beta$-CD at $c a$. $304{ }^{\circ} \mathrm{C}$. In contrast, the decomposition temperature of the transpolydatin- $\beta-\mathrm{CD}$, trans-polydatin-Me- $\beta-\mathrm{CD}$, and trans-polydatinHP- $\beta$-CD inclusion complex is ca. $281{ }^{\circ} \mathrm{C}, 288{ }^{\circ} \mathrm{C}$, and $298{ }^{\circ} \mathrm{C}$. These results confirm the formation of the trans-polydatin-CDs inclusion complexes. Furthermore, the thermal stability of trans-polydatin was enhanced after encapsulating by CDs.

DSC gave further information about the thermal properties of trans-polydatin- $\beta-\mathrm{CD}$, trans-polydatin-Me- $\beta-\mathrm{CD}$, and transpolydatin-HP- $\beta$-CD inclusion complex. As shown in Fig. 4 , transpolydatin displayed one sharp endothermic peak at $230{ }^{\circ} \mathrm{C}$. In contrast, the DSC curves of $\beta-C D, M e-\beta-C D$, and HP- $\beta-C D$ had an endothermic peak at $325{ }^{\circ} \mathrm{C}, 339{ }^{\circ} \mathrm{C}$, and $323^{\circ} \mathrm{C}$, respectively.
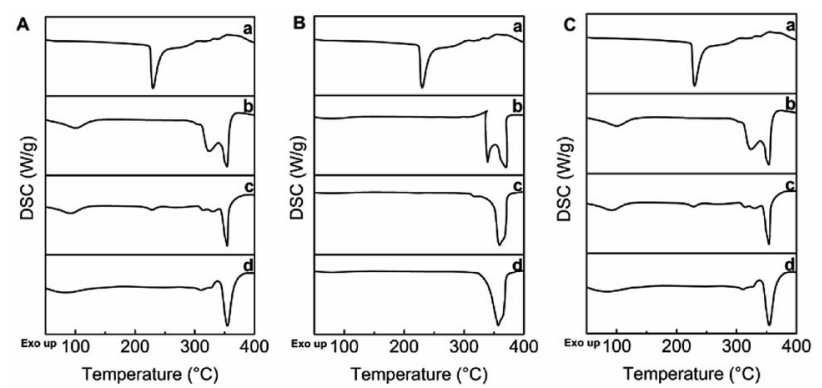

Fig. 4 DSC thermograms: (A) (a) trans-polydatin, (b) $\beta-C D$, (c) transpolydatin $/ \beta-C D$ physical mixture, (d) trans-polydatin- $\beta-C D$ inclusion complex; (B) (a) trans-polydatin, (b) Me- $\beta-C D$, (c) trans-polydatin and Me- $\beta$-CD physical mixture, (d) trans-polydatin-Me- $\beta-C D$ inclusion complex; (C) (a) trans-polydatin, (b) HP- $\beta-C D$, (c) trans-polydatin/HP$\beta-C D$ physical mixture, (d) trans-polydatin-HP- $\beta-C D$ inclusion complex.

The DSC thermogram of the physical mixture is basically a combination of two components, with the trans-polydatin peaks only faintly observable due to the lower proportions it had in the physical mixture. However, in the DSC curves of transpolydatin- $\beta-\mathrm{CD}$, trans-polydatin-Me- $\beta-\mathrm{CD}$, and trans-polydatinHP- $\beta$-CD inclusion complex, the endothermic peaks were shifted to $354{ }^{\circ} \mathrm{C}, 357^{\circ} \mathrm{C}$, and $354{ }^{\circ} \mathrm{C}$, suggesting that an inclusion structure was formed between the host guest molecules. These results further confirm the formation of inclusion complex between trans-polydatin and CDs. ${ }^{34}$

\section{SEM studies}

SEM is a qualitative method used to study the structural aspects of the materials. ${ }^{35,36}$ Fig. 5 shows the SEM photographs of transpolydatin, HP- $\beta-C D$, their physical mixture, and inclusion complex. Pure trans-polydatin existed in columnar crystal with medium dimensions and HP- $\beta$-CD appears as a spherical shape with cavity structures. The physical mixture of trans-polydatin with CDs revealed the characteristic crystals of trans-polydatin and the spheres of HP- $\beta$-CD both existed separately, indicating the two components exist in their original individual forms. In contrast, the trans-polydatin-CDs inclusion complex appeared as plate-like structure crystal and was quite different from the sizes and shapes of trans-polydatin and CDs, which confirms the formation of the inclusion complex between trans-polydatin and HP- $\beta$-CD. In similar test, the trans-polydatin- $\beta$-CD and trans-polydatin-Me- $\beta$-CD appeared to be quite different from the sizes and shapes of $\beta-\mathrm{CD}, \mathrm{Me}-\beta-\mathrm{CD}$ and trans-polydatin, respectively, which are a strong indication of inclusion complex formation (see in the ESI Fig. S4 and S5†).

\section{${ }^{1}$ H-NMR and 2D NMR}

Further evidence supporting the formation of the inclusion complex was obtained by ${ }^{1} \mathrm{H}-\mathrm{NMR}$, which has proved to be the most direct evidence in explaining the host-guest interaction of CDs and guest molecules. ${ }^{37,38}$ We measured the ${ }^{1} \mathrm{H}-\mathrm{NMR}$ spectra of the CDs and the inclusion complexes of trans-polydatin- $\beta-\mathrm{CD}$, trans-polydatin-Me- $\beta$-CD, and trans-polydatin-HP- $\beta-\mathrm{CD}$ in $\mathrm{D}_{2} \mathrm{O}$ 


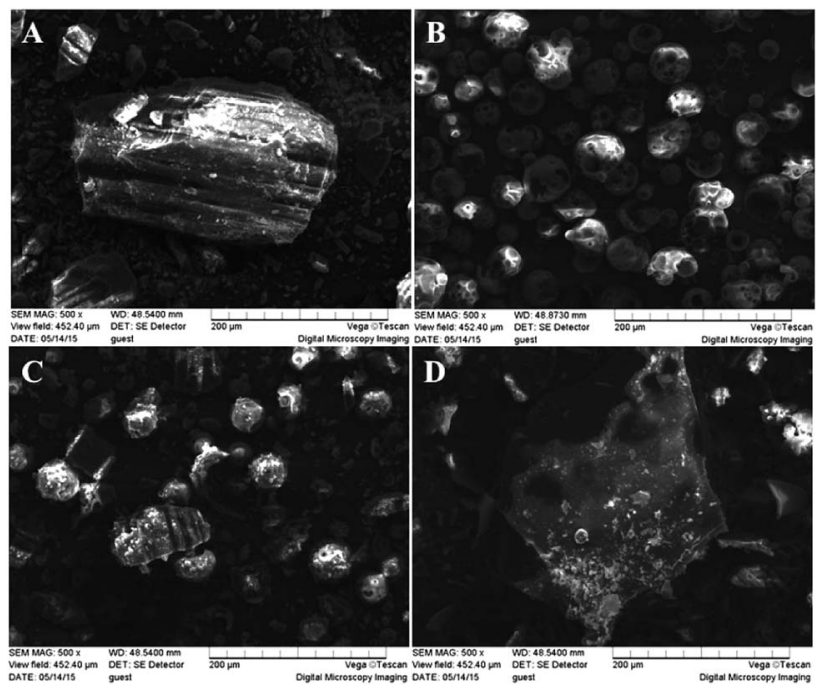

Fig. 5 Scanning electron microphotographs: (A) trans-polydatin, (B) HP- $\beta-C D,(C)$ trans-polydatin/HP- $\beta-C D$ physical mixture, (D) transpolydatin-HP- $\beta-C D$ inclusion complex.

(see ESI Fig. S6-S11 $\dagger$ ). The ${ }^{1} \mathrm{H}-\mathrm{NMR}$ spectra of the inclusion complexes showed all of the expected proton signals of transpolydatin and CDs, in agreement with the formation of the inclusion complex.

To understand the detailed inclusion fashion of trans-polydatin- $\beta$-CD, trans-polydatin-Me- $\beta-\mathrm{CD}$, and trans-polydatin-HP- $\beta$ CD, 2D ROESY NMR spectra were also measured. As shown in Fig. 6, the 2D ROESY NMR spectrum of trans-polydatin-HP- $\beta$-CD showed strong correlation signals between the inner $\mathrm{H}-3$ and $\mathrm{H}$ 5 protons of the HP- $\beta$-CD and the trans-polydatin protons. The spectrum exhibited strong correlation signals between the $\mathrm{H}-3$ protons of the HP- $\beta-C D$ and the $\mathrm{H}-7,8$ of trans-polydatin. Meanwhile, the correlation signals were also observed between $\mathrm{H}-5$ protons of the HP- $\beta$-CD and $\mathrm{H}-11,13$ protons of trans-polydatin. However, the spectrum did not show any significant correlation signals between the H-5 proton of the HP- $\beta-C D$ and

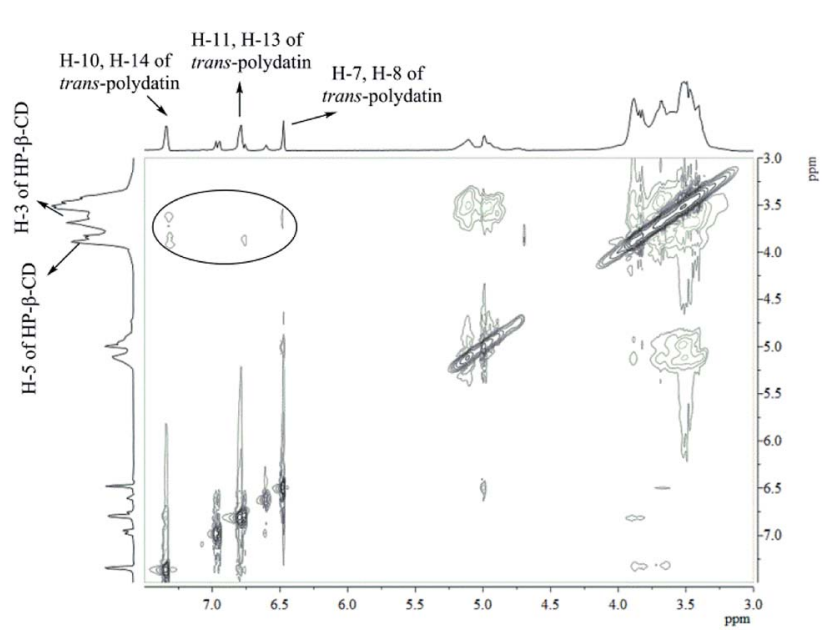

Fig. 6 ROESY spectrum of the trans-polydatin-HP- $\beta-C D$ inclusion complex in $\mathrm{D}_{2} \mathrm{O}$ at $25^{\circ} \mathrm{C}$.

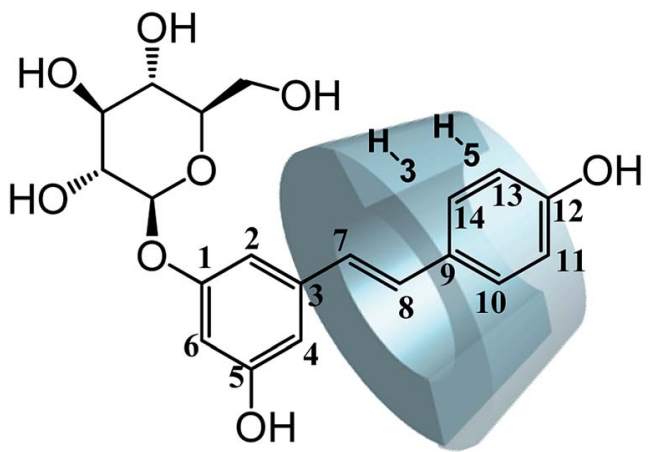

Fig. 7 Possible inclusion mode of the trans-polydatin-HP- $\beta-C D$ inclusion complex.

the $\mathrm{H}-7,8$ protons of trans-polydatin. These data indicate that the HP- $\beta$-CD selectively includes the trans-polydatin from the wide rim side to form the inclusion complex. It was also shown that trans-polydatin should be encapsulated in the $\beta$-CD and Me- $\beta$-CD cavities in a similar way (see ESI Fig. S12 and $13 \dagger$ ). A study reported by Zhang et al. ${ }^{24}$ indicated that the formation of the inclusion complex between trans-polydatin with $\beta$-CD and $\gamma$ CD also occurred through the aromatic ring of trans-polydatin into the CDs cavity, which is similar to our findings. Based on these observations, together with the 1:1 stoichiometry, we deduced the possible inclusion modes of trans-polydatin with CDs, as illustrated in Fig. 7.

\section{Photostability in free and complex form}

In order to examine the photostability of the inclusion complexes, the solutions of trans-polydatin $(p<0.05)$ and transpolydatin-CDs $(p<0.05)$ at the concentration of $6 \times$ $10^{-5} \mathrm{~mol} \mathrm{~L}^{-1}$ were exposed to UV light. Monitoring the reduction of trans-polydatin concentration during the irradiating time it was obtained using UV-vis method. The effect of CDs on the photostability of trans-polydatin is illustrated in Fig. 8. After comparison of the photoisomerization profiles obtained for trans-polydatin and its complexes, it can be concluded that the CDs had a significant effect on the rate of trans-polydatin photoisomerization. After $180 \mathrm{~min}$ irradiation, the content of transpolydatin was decreased for $54 \%$, while the content of transpolydatin in trans-polydatin- $\beta-\mathrm{CD}$, trans-polydatin-Me- $\beta-\mathrm{CD}$, and trans-polydatin-HP- $\beta$-CD complexes was decreased for $42 \%$, $36 \%$ and $28 \%$, respectively. These results showed that the complexation of trans-polydatin with CDs led to an increase in the trans-polydatin photostability during the exposure to UV radiation. This finding is consistent with previous study. ${ }^{39,40}$ Therefore, trans-polydatin was highly protected against photoisomerization by inclusion complexation.

\section{Antioxidant activity of polydatin in free and complex form}

The reducing power is a sensible evidence for the antioxidant activity of an extract or a compound. ${ }^{\mathbf{4 1 , 4 2}}$ In the reducing power assay, the $\mathrm{Fe}^{3+}$ /ferricyanide complex was reduced to the $\mathrm{Fe}^{2+}$ ions by the presence of antioxidants. The reducing reaction can be monitored spectrophotometrically by recording the 


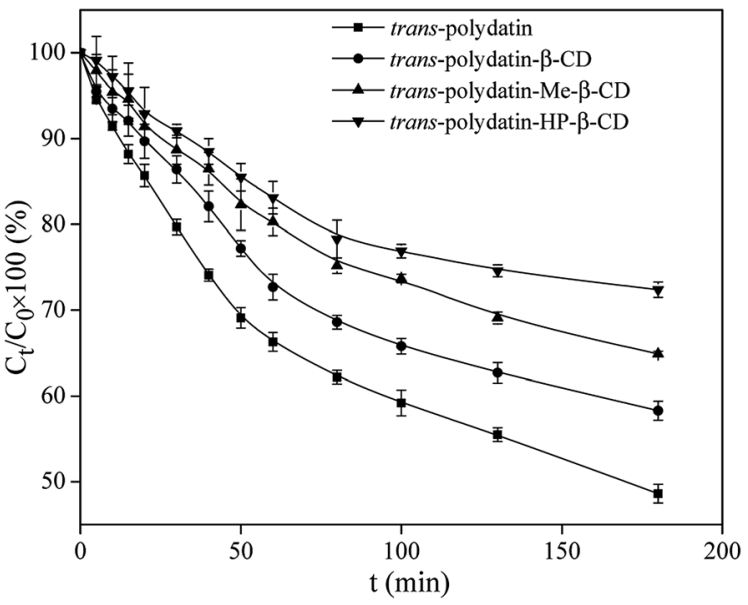

Fig. 8 The photoisomerization of trans-polydatin in the absence and presence of $\beta-C D, M e-\beta-C D$ and $H P-\beta-C D$.

absorbance at $700 \mathrm{~nm}$. According to the mentioned principle, the higher absorbance value at $700 \mathrm{~nm}$ represent a stronger reducing power of the reaction compound.

Fig. 9 shows a comparison of the reducing power values of trans-polydatin $(p<0.05)$, trans-polydatin- $\beta$-CD $(p<0.05)$, transpolydatin-Me- $\beta$-CD $(p<0.05)$, and trans-polydatin-HP- $\beta-\mathrm{CD}(p<$ 0.05 ) complexes. The reducing powers values of the four samples were all enhanced along with the increasing concentration among the tested range, but the reducing power values of the three inclusion complexes were stronger than that native trans-polydatin. Since this assay was carried out in an aqueous solution, the hydrophobic and assembled trans-polydatin molecules could not fully close and react with the $\mathrm{Fe}^{3+} /$ ferricyanide ions in the test. However, the complexation made the trans-polydatin was totally dissolved in water. Thus, the reducing power of the three inclusion complexes was stronger than that of native trans-polydatin. Additionally, the reducing power order of the different inclusion complex is trans-polydatin-HP- $\beta$-CD $>$ trans-polydatin-Me- $\beta-\mathrm{CD}>$ trans-polydatin- $\beta$ CD.

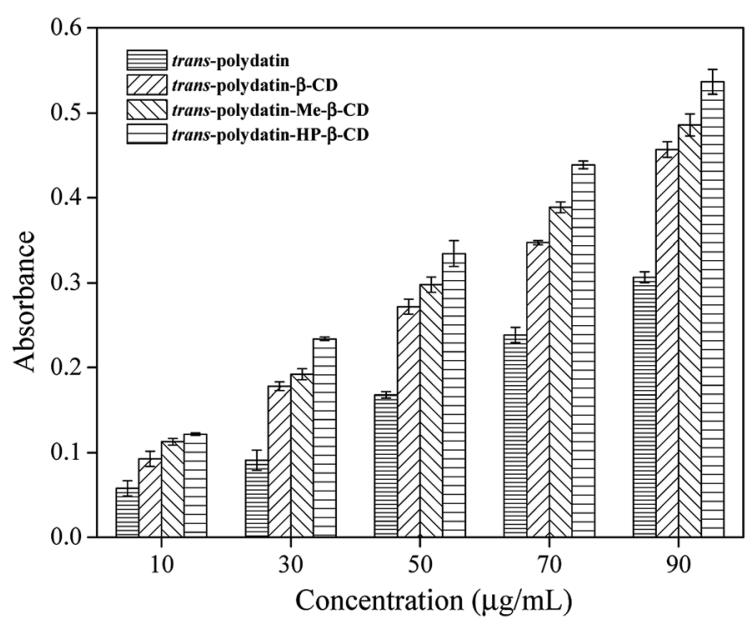

Fig. 9 Reduce powers of trans-polydatin, trans-polydatin- $\beta-C D$, trans-polydatin-Me- $\beta-C D$, and trans-polydatin-HP- $\beta-C D$ inclusion complex.

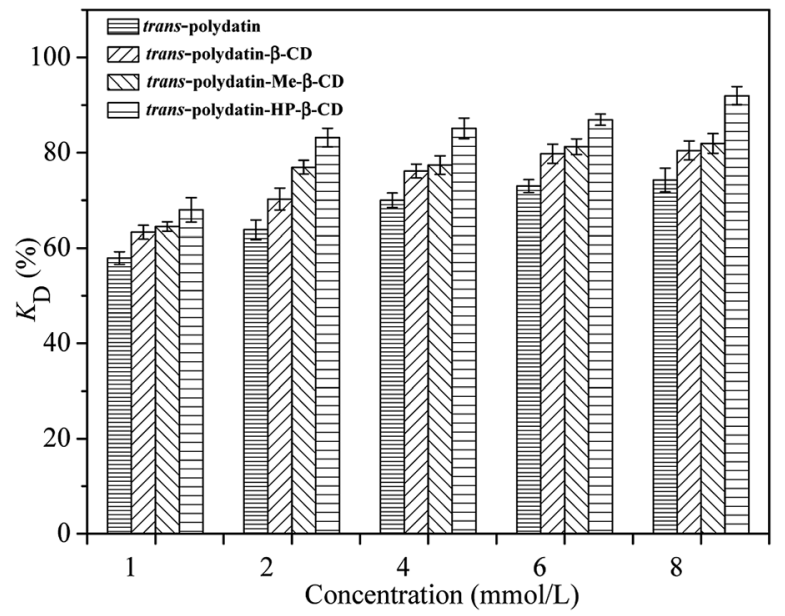

Fig. 10 DPPH radical scavenging activities of the trans-polydatin, trans-polydatin- $\beta-C D$, trans-polydatin-Me- $\beta-C D$, and trans-polydatin-HP- $\beta-C D$ inclusion complex.

Fig. 10 shows a comparison of the DPPH radical-scavenging activity of trans-polydatin $(p<0.05)$, trans-polydatin- $\beta$ - $\mathrm{CD}(p<$ $0.05)$, trans-polydatin-Me- $\beta-\mathrm{CD}(p<0.05)$, and trans-polydatinHP- $\beta$-CD complexes $(p<0.05)$. As can be seen, after complexation with CDs, the scavenging ability of trans-polydatin increased significantly. The order is trans-polydatin-HP- $\beta$-CD > trans-polydatin-Me- $\beta$-CD > trans-polydatin- $\beta-\mathrm{CD}$, which indicates that the trans-polydatin-CDs complexes have stronger DPPH radical-scavenging ability than native trans-polydatin. The DPPH scavenging ability of the antioxidant is closely related to its hydrogen-donating ability. ${ }^{434}$ The increasing DPPH scavenging ability of trans-polydatin could be attributed to the enhancement of its hydrogen-donating ability, caused by the complexation of CDs. When trans-polydatin is complexed with CDs, one or more intermolecular hydrogen bonds form between trans-polydatin and the CDs. This weakens the intramolecular hydrogen bonds of trans-polydatin. Ultimately, the hydrogen-donating ability of trans-polydatin is improved. The stronger interaction between trans-polydatin and HP- $\beta-C D$ weakened the covalent bonds between hydrogen and oxygen in the hydroxyl groups, which in turn improved the hydrogen donation of the hydroxyl groups of trans-polydatin. In contrast, the multiple methyl group substitutions of Me- $\beta-\mathrm{CD}$ impaired the hydrogen-bonding interaction between transpolydatin and Me- $\beta$-CD. This is unfavorable to the hydrogendonating ability of trans-polydatin. Ultimately, the DPPH scavenging ability of trans-polydatin-HP- $\beta$-CD is stronger than that of trans-polydatin-Me- $\beta$-CD, which is consistent with the binding ability of the three CDs. Therefore, we can conclude that the antioxidant property of trans-polydatin-CDs is closely related their mode of binding. Additionally, the effective stabilization of radical species in the CDs cavity also plays an important role in the scavenging activity of DPPH free radical. The similar results were also found for canthaxanthin and canthaxanthin/HP- $\beta$-CD complex. ${ }^{45,46}$ 


\section{Conclusions}

The inclusion complexes of trans-polydatin with $\beta$-CD, Me- $\beta$-CD and HP- $\beta$-CD were prepared and characterized by phasesolubility, XRD, DSC, SEM, ${ }^{1} \mathrm{H}$ NMR, 2D NMR, photostability and antioxidant studies. The phase-solubility, XRD, DSC, SEM, ${ }^{1} \mathrm{H}$ NMR and 2D NMR studies confirmed that trans-polydatin can form the inclusion complexes with three kinds of CDs, and the ratio between the host-guest molecules is $1: 1$. Furthermore, the solubility and thermal stability of trans-polydatin was improved due to the formation of inclusion complex. The ${ }^{1} \mathrm{H}$ NMR and 2D ROESY analyses showed that the aromatic ring of trans-polydatin was the part that inserted into the cavity of CDs, thus forming an inclusion complex. The photostability of trans-polydatin was enhanced after encapsulating by CDs. Antioxidant activity studies showed that the antioxidant performance of the inclusion complexes was better than the native trans-polydatin, and trans-polydatin-HP- $\beta-C D$ inclusion complex was the most effective form. Given the easy preparation and environmentally friendly process of trans-polydatin-CDs inclusion complexes, it is a promising way to design a novel formulation of trans-polydatin for its drug bioavailability or phytochemical preparations.

\section{Conflicts of interest}

There are no conflicts to declare.

\section{Acknowledgements}

This work is financially supported by the National Natural Science Foundation of China (31501445).

\section{References}

1 W. W. Xing, J. Z. Wu, M. Jia, J. Du, H. Zhang and L. P. Qin, Biomed. Pharmacother., 2009, 63, 457-462.

2 L. Yang, Y. Sun and G. Huang, Int. J. Biol. Macromol., 2018, 111, 780 .

3 H. Zhang, Pharm. Biol., 2013, 51, 1347-1354.

4 C. W. Shan, Acta Pharm. Sin., 1988, 23, 394-396.

5 H. Li, B. Shi, Y. Li and F. Yin, J. Biochem. Mol. Toxicol., 2017, 31, e21900.

6 Z. Kerem, I. Bilkis, M. A. Flaishman and L. Sivan, J. Agric. Food Chem., 2006, 54, 1243-1247.

7 H. Qiao, H. Chen, Y. Dong, H. Ma, G. Zhao, F. Tang and Z. Li, Oxid. Med. Cell. Longevity, 2016, 2016, 5139458.

8 X. Xie, J. Peng, K. Huang, J. Huang, X. Shen, P. Liu and H. Huang, Mol. Cell. Endocrinol., 2012, 362, 183-193.

9 A. A. Bertelli, F. Ferrara, G. Diana, A. Fulgenzi, M. Corsi, W. Ponti, M. E. Ferrero and A. Bertelli, Int. J. Tissue React., 1999, 21, 93-104.

10 S. Fabris, F. Momo, G. Ravagnan and R. Stevanato, Biophys. Chem., 2008, 135, 76-83.

11 S. Mathew, M. Hedström and P. Adlercreutz, Process Biochem., 2012, 47, 528-532.

12 J. Szejtli, Chem. Rev., 1998, 98, 1743-1754.
13 J. G. Galvão, V. F. Silva, S. G. Ferreira, F. R. M. França, D. A. Santos, L. S. Freitas, P. B. Alves, A. A. S. Araújo, S. C. H. Cavalcanti and R. S. Nunes, Thermochim. Acta, 2015, 608, 14-19.

14 D. Leonardi, M. E. Bombardiere and C. J. Salomon, Int. J. Biol. Macromol., 2013, 62, 543-548.

15 O. I. Maloletkina, K. A. Markossian, R. A. Asryants, P. I. Semenyuk, V. F. Makeeva and B. I. Kurganov, Int. J. Biol. Macromol., 2010, 46, 487-492.

16 J. Wang, W. Li, H. Yu, Z. U. Abdin, Y. Chen, Q. Chen, W. Zhou, H. Zhang and C. Xiao, Int. J. Biol. Macromol., 2016, 88, 333-344.

17 Y. Wei, J. Zhang, Y. Zhou, W. Bei, Y. Li, Q. Yuan and H. Liang, Carbohydr. Polym., 2017, 159, 152.

18 D. Duchêne, A. Bochot and T. Loftsson, STP Pharma Prat., 2009, 19, 15-27.

19 H. M. C. Marques, A. C. Figueiredo and M. G. Miguel, Flavour Fragrance J., 2010, 25, 313-326.

20 J. Carlstedt, A. González-Pérez, M. Alatorre-Meda, R. S. Dias and B. Lindman, Int. J. Biol. Macromol., 2010, 46, 153-158.

21 M. Terauchi, T. Inada, A. Tonegawa, A. Tamura, S. Yamaguchi, K. Harada and N. Yui, Int. J. Biol. Macromol., 2016, 93, 1492-1498.

22 S. An, J. He, L. Sun, D. Ren and Y. Ban, J. Mol. Struct., 2013, 1037, 9-14.

23 B. Liu, Y. Li, H. Xiao, Y. Liu, H. Mo, H. Ma and G. Liang, J. Food Sci., 2015, 80, C1156-C1161.

24 J. Q. Zhang, K. M. Jiang, X. G. Xie, Y. Jin and J. Lin, J. Mol. Liq., 2016, 219, 592-598.

25 X. H. Li, Z. Y. Jin and J. Wang, Food Chem., 2007, 103, 461466.

26 T. Higuchi and K. A. Connors, Adv. Anal. Chem. Instrum., 1965, 4, 117-212.

27 S. Ma, Z. Zhao and P. Liu, J. Food Meas. Charact., 2018, 1-11.

28 T. Hatano, H. Kagawa, T. Yasuhara and T. Okuda, Chem. Pharm. Bull., 1988, 36, 2090-2097.

29 S. E. Çelik, M. Özyürek, K. Güçlü and R. Apak, J. Inclusion Phenom. Macrocyclic Chem., 2015, 83, 309-319.

30 Q. F. Zhang, H. C. Nie, X. C. Shangguang, Z. P. Yin, G. D. Zheng and J. G. Chen, J. Agric. Food Chem., 2013, 61, 151-156.

31 Z. Aytac, S. Ipek, E. Durgun and T. Uyar, J. Mater. Sci., 2018, 53, 1527-1539.

32 A. Celebioglu and T. Uyar, J. Agric. Food Chem., 2017, 65, 5404.

33 A. M. Dias, S. D. G. Da, Â. M. Fiorentini, V. Z. Pinto, L. T. Lim, R. Z. E. Da and D. Arg, Int. J. Biol. Macromol., 2017, 104, 874.

34 L. P. N. De, S. D. D. Da, V. G. Costa, D. Q. Falcão and D. L. A. Kg, Food Chem., 2016, 208, 124-131.

35 T. Pralhad and K. Rajendrakumar, J. Pharm. Biomed. Anal., 2004, 34, 333-339.

36 S. Zidane, A. Maiza, H. Bouleghlem, W. Herizi and S. Dahmani, 2016.

37 H. J. Schneider, F. Hacket, V. Rüdiger and H. Ikeda, Chem. Rev., 1998, 98, 1755.

38 K. Srinivasan and T. Stalin, Spectrochim. Acta, Part A, 2014, 130, 105-115. 
39 S. Dong, Y. Huang, R. Zhang, Z. Lian, S. Wang and Y. Liu, Eur. J. Lipid Sci. Technol., 2014, 116, 978-986.

40 I. Savic-Gajic, I. M. Savic, V. D. Nikolic, L. B. Nikolic, M. M. Popsavin and A. J. Kapor, J. Inclusion Phenom. Macrocyclic Chem., 2016, 86, 1-11.

41 K. L. Yap, X. Liu, J. C. Thenmozhiyal and P. C. Ho, Eur. J. Pharm. Sci., 2005, 25, 49-56.

42 B. Liu, M. A. Yuxiang, C. Yuan, S. U. Caijuan, H. U. Lizhi and J. Wang, J. Food Biochem., 2012, 36, 634-641.
43 A. Corciova, C. Ciobanu, A. Poiata, C. Mircea, A. Nicolescu, M. Drobota, C. D. Varganici, T. Pinteala and N. Marangoci, Dig. J. Nanomater. Biostruct., 2014, 9, 1623-1637.

44 Q. Li, H. Pu, P. Tang, B. Tang, Q. Sun and H. Li, Food Chem., 2017, 245, 1062-1069.

45 M. Strazisar, S. Andrensek and A. Smidovnik, Food Chem., 2008, 110, 636-642.

46 C. Jullian, T. Orosteguis, F. P'erez-Cruz, P. Sanchez, F. Mendizabal and C. Olea-Azar, Spectrochim. Acta, Part A, 2008, 71, 269-275. 